\section{Weiterer Beleg für den Nutzen von Abirateron bei metastasiertem Prostatakarzinom}

\author{
Die frühe Gabe von Abirateron in der Therapie von Patienten mit metasta- \\ siertem Prostatakarzinom verlängert die Überlebenszeiten signifikant, wie \\ die Ergebnisse der LATITUDE-Studie zeigen.
}

A n der doppelblinden, randomisierten und placebokontrollierten Phase-III-Studie LATITUDE waren 1.199 Männer mit neu diagnostiziertem, metastasiertem und hormonempfindlichem Prostatakrebs beteiligt. Sie erhielten randomisiert $1.000 \mathrm{mg}$ Abirateron plus $5 \mathrm{mg}$ Prednison zusätzlich zur Androgendeprivationstherapie (ADT) oder die ADT plus Placebo.

Das Gesamtüberleben nach median 30,4 Monaten war in der AbirateronGruppe signifikant länger als bei den Männern mit ADT plus Placebo. Das mediane Überleben betrug bei Letzteren 34,7 Monate, bei Ersteren war der Medianwert noch nicht erreicht (Hazard Ratio [HR] für Tod 0,62; 95\%-Konfidenz- intervall [95\%-KI] 0,51-0,76; $\mathrm{p}<0,001)$. Auch das radiologisch progressionsfreie Überleben verlängerte sich (33,0 vs. 14,8 Monate). Für den Endpunkt Progression oder Tod reduzierte Abirateron das Risiko um $53 \%$ (HR 0,47; $95 \%$-KI 0,390,55; $\mathrm{p}<0,001)$.

In allen sekundären Endpunkten darunter Schmerzprogression, nötige Folgetherapie, Beginn der Chemotherapie, PSA-Anstieg und Komplikationen am Skelett - schnitten die Patienten unter Abirateron besser ab. Dies führte schließlich zur Empfehlung des Monitoringkomitees, die Verblindung aufzuheben und ein Crossover zu Abirateron für jene Patienten zu ermöglichen, die bisher Placebo bekommen hatten.
$63 \%$ der Patienten in der Gruppe mit Abirateron-Gabe und $48 \%$ jener in der ADT-Placebo-Gruppe entwickelten Toxizitäten vom Grad 3/4. Hypertonien und Hypokaliämien traten unter Abirateron-Prednison-ADT häufiger auf als unter ADT plus Placebo. Bei 12\% (Abirateron) bzw. $10 \%$ (Placebo) der Patienten musste die Therapie nebenwirkungsbedingt abgebrochen werden, $5 \%$ bzw. $4 \%$ der Patienten starben.

Fazit: Die Resultate von LATITUDE zeigen ein beachtliches therapeutisches Potenzial der Zugabe von Abirateron zur ADT bei Männern mit metastasiertem Prostatakrebs. Bisher bildet in diesen Fällen die Kombination von ADT mit Docetaxel den Therapiestandard. Das könnte sich durch die guten Resultate, die in dieser und der STAMPEDE-Studie mit Abirateron erzielt worden sind, aber durchaus ändern. Robert Bublak

Fizazi K et al. Abiraterone plus Prednisone in Metastatic, Castration-Sensitive Prostate Cancer. N Engl J Med. 2017;377(4):352-60.

\section{Früher Einsatz von Abirateron bringt Vorteile für Patienten mit metastasiertem Prostatakarzinom}

\author{
Patienten mit lokal fortgeschrittenem, metastasiertem oder rezidiviertem \\ Prostatakarzinom leben länger, wenn sie Abirateron zusätzlich zur Androgen- \\ deprivation erhalten.
}

\begin{abstract}
A birateron ist ein steroidaler Inhibitor der Androgen-Biosynthese. Die Verbindung hemmt selektiv das Enzym CYP17 und unterdrückt die Synthese von Androgen in Hoden, Nebennieren und Tumorgewebe. In der Kombination mit Prednison oder Prednisolon ist Abirateron zugelassen zur Behandlung metastasierter, kastrationsresistenter Prostatakarzinome, bei denen eine Androgenentzugstherapie versagt hat und eine Chemotherapie noch nicht klinisch indiziert ist oder die während oder nach einer Docetaxel-haltigen Chemotherapie progredient sind.

Daten der STAMPEDE-Studie haben nun gezeigt, dass $1.000 \mathrm{mg}$ Abirateron zusammen mit $5 \mathrm{mg}$ Prednisolon, bereits
\end{abstract}

zusätzlich zur Androgendeprivationstherapie (ADT) gegeben, einen erheblichen Nutzen nach sich zieht. In STAMPEDE werden verschiedene Therapieregimes auf ihre Erstlinientauglichkeit in Kombination mit der ADT getestet. Teilnehmer waren im Fall von Abirateron 1.917 Patienten mit neu diagnostiziertem, lokal fortgeschrittenem oder bereits metastasiertem Hochrisiko-Prostatakarzinom bzw. Hochrisikorezidiven.

Nach median 40 Monaten waren 184 Männer in der Kombinationsgruppe und 267 in der ADT-Placebo-Gruppe verstorben. Die Mortalitätsreduktion betrug $37 \%$ zugunsten der Abirateron-Kombination. Für Patienten mit nicht metastasierten Tumoren lag der Nutzen bei $25 \%$, für Männer mit Metastasen bei 39\%. Bei 248 Männern unter Abirateron-Prednisolon-ADT wuchs das Karzinom weiter, verglichen mit 535 unter ADT-Placebo. Das Risiko für ein Therapieversagen sank unter der Kombination um 71 \%: um 79\% für Männer ohne und um 69\% für Männer mit Metastasen. Nebenwirkungen vom Grad 3-5 betrafen $47 \%$ der Patienten in der Abirateron- und 33\% in der Placebo-Gruppe. Von Grad-5-Nebenwirkungen waren 9 Patienten im Abirateronund $3 \mathrm{im}$ Placebo-Arm betroffen.

Fazit: Die Resultate von STAMPEDE und LATITUDE dürften die Diskussion um die optimale Erstlinientherapie von Männern mit lokal fortgeschrittenem oder metastasiertem Prostatakrebs befeuern. Experten warten nun auf die Resultate weiterer Studien, die einen Vergleich des Nutzens von Abirateron und Docetaxel erlauben. Robert Bublak

James ND et al. Abiraterone for Prostate Cancer Not Previously Treated with Hormone Therapy. N Engl J Med. 2017;377(4):338-51. 